\title{
Estudio del estado nutricional y de la prevalencia de exceso de peso en adolescentes de localidades extremeñas de diferentes tamaños
}

\section{A study of the nutritional status and prevalence of overweight among adolescents from different size towns in Extremadura}

\begin{abstract}
RESUMEN
En este trabajo se determina el estado nutricional de adolescentes de Extremadura y la prevalencia de sobrepeso y obesidad en función del tamaño de la localidad de procedencia. Se realizó un estudio con 816 estudiantes de Educación Secundaria Obligatoria y Bachillerato, de ambos sexos, con edades de 13 a 18 años. Los centros educativos pertenecían a municipios de una Región española, Extremadura, de diferentes tamaños (de menos de 5.000 a más de 60.000 habitantes). Se recogieron datos antropométricos y, siguiendo criterios nacionales e internacionales, se determinaron los porcentajes de sobrepeso y obesidad en función del tamaño de las localidades. Los alumnos de localidades rurales (menores de 5.000 habitantes) tienden a presentar valores de diferentes parámetros antropométricos que se relacionan con un menor desarrollo corporal. Aunque hubo variaciones en la prevalecía de sobrepeso y obesidad en función de los criterios utilizados, las localidades de más de 60.000 habitantes presentaron los mayores porcentajes medios de exceso de peso y las rurales, los menores.

Palabras clave: Adolescentes extremeños; Obesidad; Sobrepeso; Valoración antropométrica; Zonas rurales.
\end{abstract}

\footnotetext{
ABSTRACT

The aim of this study was to determine the nutritional status and estimate the prevalence of overweight and obesity among adolescents from different size towns in the Extremadura Region of Spain. A sample of 816 secondary school students of both sexes between 13 and 18 years of age were evaluated. The educative centers selected belonged to different size towns (populations between $<5,000$ and $>60,000$ inhabitants). Different anthropometric measures were taken. The percentage of teenagers with overweight and obesity was calculated following national and international standards and compared by town size. Students from rural towns, <5,000 inhabitants, had the lowest values in some of the anthropometric measures; results that are associated with a lower body development. There were differences in the prevalence of overweight and obesity based on the criteria used, but, in general, adolescents from larger towns (>60,000 inhabitants) showed the highest percentages of
}

Jacinta Fernández-Cabrera ${ }^{1}$, Emilio Aranda', María de Guía Córdoba ${ }^{7}$, Francisco Pérez-Nevado ${ }^{7}$

1. Área de Nutrición y Bromatología, Departamento de Producción Animal y Ciencia de los Alimentos, Escuela de Ingenierías Agrarias, Universidad de Extremadura. Badajoz Spain.

Este trabajo fue recibido el 06 de diciembre de 2017. Aceptado con modificaciones: 04 de junio de 2018. Aceptado para ser publicado: 06 de agosto de 2018.

overweight, whereas rural populations showed the lowest percentages.

Key words: Anthropometry; Extremadura's adolescents; obesity; Overweight; Rural populations.

\section{INTRODUCCIÓN}

El estado nutricional de los adolescentes ha sido una constante preocupación en los últimos tiempos; es bien sabido que éste es un periodo clave que afecta a las condiciones de salud en la edad adulta ${ }^{1,2,3}$. Desde hace años se vienen apreciando cambios en los patrones alimenticios de la población y en la práctica de actividad física, que, en las últimas dos décadas ha tenido como consecuencia un incremento en los niveles de obesidad y sobrepeso, tanto en 
países desarrollados como otros en vías de desarrollo ${ }^{4,5,6,7}$.

En España se han realizado diversos estudios que demuestran la alta prevalencia del sobrepeso y la obesidad en niños y adolescentes ${ }^{8,9,10,11,12}$. Según datos recogidos en la Encuesta Nacional de Salud de España 2017, en torno al $28 \%$ de niños y adolescentes tienen sobrepeso y el $10 \%$ son obesos ${ }^{13}$. Hoy día, la obesidad es considerada una enfermedad crónica, que se ha relacionado con un aumento en el riesgo de padecer diabetes mellitus tipo 2, hipertensión, patología cardiovascular o cáncer ${ }^{5,11}$. Además de otros factores, según trabajos previos ${ }^{1,14,15,16}$, la prevalencia de sobrepeso y obesidad también parece estar relacionadas con el nivel socioeconómico y de estudios, y con el tamaño y localización del municipio donde residan.

A la hora de definir sobrepeso y obesidad en la infancia y la adolescencia, no hay un criterio único y consensuado para establecer sobrepeso y obesidad a partir del índice de masa corporal (IMC), utilizándose diferentes estándares nacionales e internacionales ${ }^{17,18,19}$, cada uno con ventajas e inconvenientes al basarse en diferentes criterios para su estimación. A nivel internacional se emplean los criterios de Cole ${ }^{17}$; en Estados Unidos se suelen utilizar las tablas desarrolladas por los Centers for Disease Control and Prevention $(C D C)^{18}$, mientras que en España se utilizan mayoritariamente las curvas y tablas de crecimiento de la Fundación Faustino Orbegozo ${ }^{19}$.

No hay muchos estudios que analicen las diferencias en el estado nutricional de adolescentes españoles y la prevalencia de sobrepeso y obesidad teniendo como base del estudio si los mismos residen en el ámbito rural o urbano. Por ello, el objetivo del presente trabajo plantea analizar el estado nutricional y la prevalencia de exceso de peso de adolescentes extremeños en función del tamaño de la localidad de procedencia, comparando la prevalencia de sobrepeso y obesidad al utilizar diferentes criterios, tanto nacionales como internacionales.

\section{MATERIAL Y MÉTODOS Muestra Poblacional}

Se realizó un estudio descriptivo transversal, con una muestra poblacional de 816 adolescentes con edades entre los 13 y 18 años de edad, 450 chicos y 366 chicas. De ellos, 529 estudiantes cursaban $2^{\circ}$ de ESO (Educación Secundaria Obligatoria) y $287,1^{\circ}$ de Bachillerato durante el curso escolar 2009/2010. Dicha muestra se obtuvo de forma aleatoria en 23 Centros de Educación Secundaria, públicos o privados/ concertados, pertenecientes a 19 localidades de las dos provincias extremeñas (Badajoz y Cáceres). Con el fin de asegurar su heterogeneidad y representatividad, así como la validez del estudio para toda la población extremeña, la muestra se escogió teniendo en cuenta criterios como estratos de población, incluyendo zonas de distinto nivel socioeconómico, rurales y no rurales, dispersión geográfica y etapas educativas. Para ello, se incluyeron centros educativos de localidades con diferentes tamaños de población, clasificándose en cinco grupos diferentes: de menos de 5.000 , de 5.000 a 10.000 , de 10.000 a 20.000 , de 20.000 a 60.000 y superiores a 60.000 habitantes respectivamente. Con el objetivo de comparar resultados con otros estudios basados en el tipo de la población de residencia, se consideraron municipios rurales aquellos con un número de habitantes inferior a los 5.000 ${ }^{15,20}$.

\section{Recogida de datos y material}

Los datos se recogieron en las instalaciones del centro educativo, en horario escolar, por personal cualificado y con formación suficiente. Para la obtención de los datos, se contó con el consentimiento informado de todos los participantes o de sus progenitores, además de la autorización de las autoridades educativas. A cada sujeto de la muestra se le determinaron las siguientes medidas antropométricas directas y estandarizadas: talla, peso, perímetro del brazo, perímetro del antebrazo, pliegue cutáneo tricipital, perímetro de la muñeca, perímetro de la cintura y perímetro de la cadera. A partir de esas medidas, y mediante la aplicación de sus correspondientes fórmulas ${ }^{21}$, se obtuvieron el área total del brazo, área muscular del brazo, área grasa del brazo, complexión, Índice de Masa Corporal o IMC y el Índice Cintura/Cadera. Todas las medidas se tomaron por triplicado utilizando material antropométrico homologado, calibrado y siguiendo la metodología recomendada por el IBP (Programa Internacional de Biología) ${ }^{22}$ e indicada en trabajos previos ${ }^{23}$.

Talla: se empleó un tallímetro SECA (Ltd., Birmingham, England) con escala métrica, desmontable de dos secciones y una longitud de $2.300 \mathrm{~mm}$ (precisión de $1 \mathrm{~mm}$ ).

Peso: se utilizó una balanza TEFAL (Bodysignal) con un peso máximo de $160 \mathrm{~kg}$ y una precisión de $100 \mathrm{~g}$.

Circunferencia de la muñeca y perímetro del brazo y antebrazo: se midieron con una cinta métrica flexible, no elástica, con un espacio sin graduar antes del cero, precisión de $1 \mathrm{~mm}$ y con una anchura de $1 \mathrm{~cm}$ y longitud de $200 \mathrm{~cm}$.

Pliegue cutáneo tricipital (PT): se midió el pliegue de la cara posterior del brazo no dominante, a nivel mesobraquial, con un lipocalibre o adipómetro digital.

Como índices indirectos se calcularon el área del brazo (Circunferencia del brazo $\left.{ }^{2}(\mathrm{~mm}) / 12,56\right)$; el Área muscular del brazo (Circunferencia del brazo $(\mathrm{mm})$ - 3,14*Pliegue tricipital $(\mathrm{mm}))^{2} / 12,56$ ); Área grasa brazo (Área del brazoárea muscular del brazo) y complexión corporal ( $r=$ Talla $(\mathrm{cm}) /$ circunferencia muñeca $(\mathrm{cm})$ ).

A partir de los datos obtenidos se realizó una determinación de la prevalencia de sobrepeso y obesidad en las localidades con diferentes tamaños de población. Se emplearon tres criterios de corte diferente para comparar los resultados de sobrepeso y obesidad al utilizar cada uno; los criterios fueron los internacionales de Cole ${ }^{17}$; los de CDC, de Eslados Unidos ${ }^{18}$ y los de España de la Fundación Faustino Orbegozo ${ }^{19}$.

Tratamiento de los datos y análisis estadístico

Una vez obtenidos, los datos fueron procesados 
empleando el paquete estadístico SPSS v. 19.0, realizándose un análisis descriptivo e inferencial. Se consideraron diferencias significativas las de probabilidad inferior al $5 \%(\mathrm{p}<0.05)$, utilizando un análisis de varianza (ANOVA), siguiendo los procedimientos de una vía y realizando un test de comparación de medias por el método Tukey. Para las estimaciones de exceso de peso por tamaño de municipio se determinó la prevalencia y el intervalo de confianza del 95\% (IC95\%).

\section{RESULTADOS}

Al analizar los resultados obtenidos por sexos para cada una de las provincias extremeñas (Tabla 1), las chicas presentaban características corporales similares en ambas, encontrándose diferencias únicamente en el área muscular del brazo. Por su parte, en los chicos había mayores diferencias, tanto en el área muscular del brazo, el área grasa del brazo, el pliegue cutáneo tricipital, el perímetro de la cintura y el perímetro de la cadera, repitiéndose los resultados a los comentados anteriormente al analizar de forma global por provincias.

En la Tabla 2 se observa que había diferencias entre chicos y chicas dependiendo del tamaño de la población de origen. Las chicas presentaron diferencias significativas para la mayoría de los parámetros, excepto para el pliegue cutáneo tricipital, la complexión, el perímetro de la muñeca

Tabla 1. Parámetros e índices antropométricos de la población adolescente por provincias.

\begin{tabular}{|c|c|c|c|}
\hline & $\begin{array}{c}\text { Badajoz } \\
(X \pm D E)\end{array}$ & $\begin{array}{c}\text { Cáceres } \\
(\mathrm{X} \pm \mathrm{DE})\end{array}$ & $\mathbf{p}$ \\
\hline CHICAS & $n=305$ & $n=145$ & \\
\hline Talla (m) & $1,59 \pm 0,07$ & $1,59 \pm 0,06$ & 0,543 \\
\hline Peso (kg) & $57,2 \pm 11,6$ & $56,4 \pm 11,0$ & 0,479 \\
\hline Perímetro del brazo $(\mathrm{cm})$ & $25,7 \pm 3,49$ & $25,5 \pm 2,87$ & 0,427 \\
\hline Área Total del Brazo $\left(\mathrm{cm}^{2}\right)$ & $53,7 \pm 15,0$ & $52,3 \pm 12,2$ & 0,328 \\
\hline Área Muscular brazo $\left(\mathrm{cm}^{2}\right)$ & $26,6 \pm 8,08$ & $24,7 \pm 5,76$ & 0,013 \\
\hline Área Grasa brazo $\left(\mathrm{cm}^{2}\right)$ & $27,1 \pm 10,2$ & $27,6 \pm 8,25$ & 0,627 \\
\hline Perímetro del antebrazo $(\mathrm{cm})$ & $22,3 \pm 2,12$ & $22,6 \pm 1,85$ & 0,147 \\
\hline Pliegue cutáneo tricipital (mm) & $24,4 \pm 7,06$ & $25,6 \pm 5,54$ & 0,127 \\
\hline Complexión (cm) & $10,6 \pm 0,78$ & $10,6 \pm 0,53$ & 0,707 \\
\hline Perímetro de la muñeca $(\mathrm{cm})$ & $15,1 \pm 1,33$ & $15,0 \pm 0,86$ & 0,690 \\
\hline Perímetro de la cintura $(\mathrm{cm})$ & $77,6 \pm 10,2$ & $78,1 \pm 9,61$ & 0,654 \\
\hline perímetro de la cadera $(\mathrm{cm})$ & $93,3 \pm 9,4$ & $92,9 \pm 9,37$ & 0,650 \\
\hline $\mathrm{IMC}\left(\mathrm{kg} / \mathrm{m}^{2}\right)$ & $22,4 \pm 4,29$ & $21,8 \pm 4,31$ & 0,202 \\
\hline Índice Cintura/Cadera (cm) & $0,83 \pm 0,08$ & $0,84 \pm 0,07$ & 0,105 \\
\hline CHICOS & $n=245$ & $n=121$ & \\
\hline Talla (m) & $1,66 \pm 0,09$ & $1,67 \pm 0,09$ & 0,407 \\
\hline Peso (kg) & $61,1 \pm 14,6$ & $62,9 \pm 12,4$ & 0,256 \\
\hline Perímetro del brazo $(\mathrm{cm})$ & $25,9 \pm 3,54$ & $26,0 \pm 3,28$ & 0,702 \\
\hline Área Total del Brazo $\left(\mathrm{cm}^{2}\right)$ & $54,2 \pm 15,5$ & $54,7 \pm 13,8$ & 0,784 \\
\hline Área Muscular brazo $\left(\mathrm{cm}^{2}\right)$ & $31,8 \pm 10,7$ & $29,6 \pm 8,51$ & 0,048 \\
\hline Área Grasa brazo $\left(\mathrm{cm}^{2}\right)$ & $22,4 \pm 9,8$ & $25,1 \pm 8,94$ & 0,012 \\
\hline Perímetro del antebrazo $(\mathrm{cm})$ & $23,7 \pm 2,33$ & $24,1 \pm 2,15$ & 0,089 \\
\hline Pliegue cutáneo tricipital (mm) & $19,5 \pm 7,43$ & $22,0 \pm 6,46$ & 0,001 \\
\hline Complexión (cm) & $10,3 \pm 0,57$ & $10,3 \pm 0,59$ & 0,598 \\
\hline Perímetro de la muñeca $(\mathrm{cm})$ & $16,1 \pm 1,04$ & $16,3 \pm 1,04$ & 0,225 \\
\hline Perímetro de la cintura $(\mathrm{cm})$ & $78,1 \pm 10,8$ & $80,4 \pm 9,2$ & 0,048 \\
\hline Perímetro de la cadera $(\mathrm{cm})$ & $91,5 \pm 10$ & $93,8 \pm 8,12$ & 0,033 \\
\hline $\mathrm{IMC}\left(\mathrm{kg} / \mathrm{m}^{2}\right)$ & $22,0 \pm 4,1$ & $22,3 \pm 4,02$ & 0,478 \\
\hline Índice Cintura/Cadera & $0,85 \pm 0,07$ & $0,86 \pm 0,06$ & 0,369 \\
\hline IMC: Índice de masa corporal. & & & \\
\hline
\end{tabular}


Tabla 2. Parámetros e índices antropométricos de la población adolescente en función del tamaño del municipio de procedencia.

\begin{tabular}{|c|c|c|c|c|c|}
\hline & $\begin{array}{l}<5.000 \\
(X \pm D E)\end{array}$ & $\begin{array}{l}5.000-10.000 \\
(X \pm D E)\end{array}$ & $\begin{array}{c}10.000-20.000 \\
(X \pm D E)\end{array}$ & $\begin{array}{c}20.000-60.000 \\
(X \pm D E)\end{array}$ & $\begin{array}{c}>60.000 \\
(X \pm D E)\end{array}$ \\
\hline Chicas & $n=120$ & $\mathbf{n}=89$ & $n=53$ & $n=75$ & $n=113$ \\
\hline Talla (m) & $1,58 \pm 0,06 a$ & $1,59 \pm 0,06 a b$ & $1,60 \pm 0,08 a b$ & $1,61 \pm 0,06 b$ & $1,60 \pm 0,06 a b$ \\
\hline Peso $(k g)$ & $53,5 \pm 10,2 \mathrm{a}$ & $57,9 \pm 12,1 \mathrm{ab}$ & $55,3 \pm 10,8 \mathrm{ab}$ & $59,7 \pm 11,5 b$ & $58,9 \pm 11,9 b$ \\
\hline Perímetro del brazo (cm) & $24,6 \pm 3,11 a$ & $26,1 \pm 3,13 b$ & $25,1 \pm 2,66 \mathrm{ab}$ & $26,0 \pm 3,34 b$ & $26,4 \pm 3,61 b$ \\
\hline Área Total del Brazo $\left(\mathrm{cm}^{2}\right)$ & $48,9 \pm 12,6 a$ & $55,0 \pm 13,7 b$ & $50,7 \pm 11,1 \mathrm{ab}$ & $54,9 \pm 14,7 b$ & $56,4 \pm 15,6 b$ \\
\hline Área Muscular brazo $\left(\mathrm{cm}^{2}\right)$ & $24,0 \pm 6,44 a$ & $25,8 \pm 5,15 a$ & $24,5 \pm 4,86 a$ & $25,9 \pm 7,81 \mathrm{ab}$ & $28,8 \pm 9,65 b$ \\
\hline Área Grasa brazo $\left(\mathrm{cm}^{2}\right)$ & $24,9 \pm 8,50 a$ & $29,2 \pm 10,0 b$ & $26,2 \pm 8,52 \mathrm{ab}$ & $29,0 \pm 9,73 a b$ & $27,6 \pm 10,3 a b$ \\
\hline Perímetro del antebrazo $(\mathrm{cm})$ & $21,9 \pm 1,99 a$ & $22,5 \pm 2,22 \mathrm{ab}$ & $22,2 \pm 1,71 \mathrm{ab}$ & $22,9 \pm 1,82 b$ & $22,7 \pm 2,15 \mathrm{ab}$ \\
\hline Pliegue cutáneo tricipital (mm) & $23,5 \pm 6,25 a$ & $26,0 \pm 6,46 a$ & $24,3 \pm 6,11 a$ & $26,1 \pm 6,47 a$ & $24,1 \pm 7,19 a$ \\
\hline Complexión (cm) & $10,7 \pm 0,76 a$ & $10,6 \pm 0,79 a$ & $10,8 \pm 0,58 a$ & $10,6 \pm 0,59 a$ & $10,6 \pm 0,71 \mathrm{a}$ \\
\hline Perímetro de la muñeca $(\mathrm{cm})$ & $14,9 \pm 1,27 a$ & $15,1 \pm 1,52 \mathrm{a}$ & $14,9 \pm 0,83 a$ & $15,2 \pm 0,79 a$ & $15,2 \pm 1,10 a$ \\
\hline Perímetro de la cintura (cm) & $75,4 \pm 8,96 a$ & $79,0 \pm 10,6 a b$ & $76,2 \pm 9,06 a b$ & $80,0 \pm 10,4 b$ & $78,7 \pm 10,2 \mathrm{ab}$ \\
\hline Perímetro de la cadera $(\mathrm{cm})$ & $89,8 \pm 9,25 a$ & $94,5 \pm 9,87 \mathrm{bc}$ & $91,4 \pm 8,45 \mathrm{ab}$ & $94,9 \pm 8,47 \mathrm{bc}$ & $95,4 \pm 9,11 \mathrm{C}$ \\
\hline IMC $\left(\mathrm{kg} / \mathrm{m}^{2}\right)$ & $21,2 \pm 3,86 a$ & $22,9 \pm 4,36 a b$ & $21,5 \pm 3,44 a b$ & $22,6 \pm 4,92 a b$ & $23,1 \pm 4,23 b$ \\
\hline Índice Cintura/Cadera & $0,84 \pm 0,07 a$ & $0,84 \pm 0,07 a$ & $0,83 \pm 0,06 a$ & $0,84 \pm 0,06 a$ & $0,82 \pm 0,06 a$ \\
\hline Chicos & $n=68$ & $n=64$ & $n=54$ & $n=78$ & $n=102$ \\
\hline Talla (m) & $1,66 \pm 0,10 \mathrm{a}$ & $1,67 \pm 0,08 \mathrm{a}$ & $1,67 \pm 0,12 \mathrm{a}$ & $1,67 \pm 0,09 a$ & $1,65 \pm 0,09 a$ \\
\hline Peso (kg) & $60,0 \pm 11,0 a$ & $60,3 \pm 11,6 a$ & $61,6 \pm 16,1 a$ & $62,4 \pm 12,9 a$ & $63,1 \pm 16,3 a$ \\
\hline Perímetro del brazo (cm) & $25,6 \pm 2,86 a$ & $25,3 \pm 2,95 a$ & $25,4 \pm 3,39 a$ & $26,1 \pm 3,18 \mathrm{a}$ & $26,6 \pm 4,20 a$ \\
\hline Área Total del Brazo $\left(\mathrm{cm}^{2}\right)$ & $52,3 \pm 11,7 a$ & $51,8 \pm 12,3 a$ & $52,4 \pm 14,4 a$ & $55,0 \pm 13,6 a$ & $57,5 \pm 18,9 a$ \\
\hline Área Muscular brazo $\left(\mathrm{cm}^{2}\right)$ & $29,8 \pm 7,16 a b$ & $30,5 \pm 7,81 \mathrm{ab}$ & $30,6 \pm 9,20 a$ & $29,2 \pm 8,20 a$ & $33,7 \pm 13,6 b$ \\
\hline Área Grasa brazo $\left(\mathrm{cm}^{2}\right)$ & $23,0 \pm 8,94 a b$ & $21,3 \pm 8,00 a$ & $21,8 \pm 8,98 \mathrm{ab}$ & $25,8 \pm 11,1 b$ & $23,6 \pm 9,73 a b$ \\
\hline Perímetro del antebrazo (cm) & $23,7 \pm 2,04 a$ & $23,4 \pm 2,28 a$ & $23,7 \pm 2,43 a$ & $24,1 \pm 2,01 a$ & $24,0 \pm 2,52 a$ \\
\hline Pliegue cutáneo tricipital (mm) & $20,4 \pm 6,97 a b$ & $18,9 \pm 6,07 a$ & $19,3 \pm 6,67 \mathrm{ab}$ & $22,7 \pm 8,43 b$ & $20,0 \pm 7,02 \mathrm{ab}$ \\
\hline Complexión (cm) & $10,3 \pm 0,55 a$ & $10,3 \pm 0,57 a$ & $10,4 \pm 0,51 a$ & $10,4 \pm 0,66 a$ & $10,2 \pm 0,56 a$ \\
\hline Perímetro de la muñeca $(\mathrm{cm})$ & $16,1 \pm 0,94 a$ & $16,2 \pm 0,95 a$ & $16,1 \pm 1,13 a$ & $16,2 \pm 1,06 a$ & $16,2 \pm 1,11 a$ \\
\hline Perímetro de la cintura $(\mathrm{cm})$ & $78,0 \pm 7,98 a$ & $77,1 \pm 9,00 \mathrm{a}$ & $78,1 \pm 10,4 a$ & $79,7 \pm 10,0 a$ & $80,4 \pm 12,5 a$ \\
\hline Perímetro de la cadera $(\mathrm{cm})$ & $91,5 \pm 7,68 a$ & $91,0 \pm 8,54 a$ & $90,9 \pm 10,2 \mathrm{a}$ & $93,1 \pm 8,31 \mathrm{a}$ & $93,6 \pm 11,3 a$ \\
\hline $\mathrm{IMC}\left(\mathrm{kg} / \mathrm{m}^{2}\right)$ & $21,4 \pm 4,06 a$ & $21,4 \pm 2,99 a$ & $21,7 \pm 3,86 a$ & $22,2 \pm 3,50 a$ & $23,0 \pm 4,96 a$ \\
\hline Índice Cintura/Cadera & $0,85 \pm 0,05 a$ & $0,85 \pm 0,05 a$ & $0,86 \pm 0,05 a$ & $0,85 \pm 0,05 a$ & $0,86 \pm 0,07 a$ \\
\hline
\end{tabular}

y el Índice cintura/cadera. Las chicas de las poblaciones rurales presentaban los valores más bajos en talla, peso, perímetro del brazo, área total del brazo, área muscular y grasa del brazo, perímetro del antebrazo, perímetro de la cintura, y de la cadera, así como para el IMC. En cuanto a los chicos, únicamente hubo diferencias, según la población analizada, en los parámetros de área muscular del brazo, área grasa del brazo y el pliegue tricipital. Los mayores valores del área muscular del brazo se dieron en poblaciones mayores de 60.000 habitantes, mientras que los valores más elevados de área grasa del brazo y pliegue tricipital se encontraron en poblaciones de 20.000 a 60.000 habitantes.

Al estudiar los parámetros e índices antropométricos de los adolescentes de poblaciones de Extremadura de más de 60.000 habitantes por edades y sexo (Tabla 3), hubo diferencias tanto en chicas y chicos con respecto a la edad en diferentes parámetros. En el caso de las chicas, el peso, el área muscular del brazo, la complexión, los perímetros de muñeca y cintura, y el IMC variaron con la edad. Por el contrario, en los chicos, los parámetros que variaron con la edad fueron la talla, el peso, el perímetro del brazo, las áreas total y muscular del brazo, y el perímetro del antebrazo.

En la Tabla 4 se muestra la prevalencia de sobrepeso y obesidad por sexo y por el tamaño del municipio de procedencia, acompañados del intervalo de confianza del 95\% obtenido. Se observaron diferencias en los parámetros estudiados en función del tamaño de la población analizada. En general, tanto los chicos como las chicas de poblaciones de menor número de habitantes presentaban los porcentajes menores de sobrepeso en ambos sexos, aunque en la cifra del 
Tabla 3. Resultado de los parámetros e índices antropométricos $\left(\mathrm{X}_{ \pm} \mathrm{DE}\right)$ de los adolescentes de poblaciones de más de 60.000 habitantes por edades y sexo.

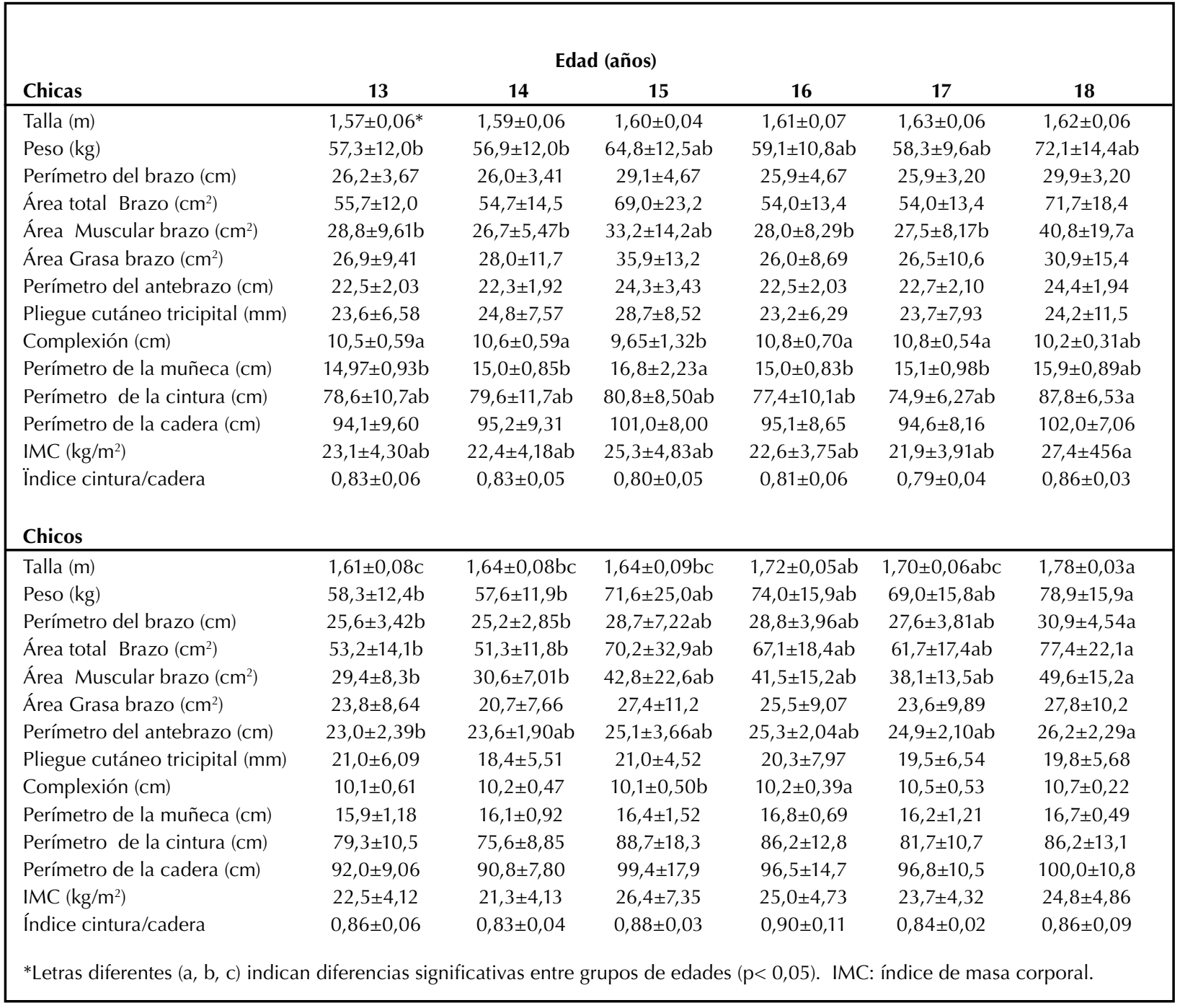

porcentaje hubo una gran variabilidad en función del criterio seguido. En cuanto a la obesidad, según la mayoría de los criterios escogidos, los chicos tenían un mayor porcentaje de obesidad que las chicas en localidades rurales y en las de más de 60.000 habitantes.

\section{DISCUSIÓN}

Los resultados obtenidos para los parámetros antropométricos por provincias indican que apenas hay diferencias entre los adolescentes de las dos provincias. Considerando los valores de referencia del índice $r$ de complexión, de Mataix ${ }^{25}$, que clasifica cada persona como de complexión pequeña ( $>10,4$ en hombre $y>11$ en mujer), mediana (entre 9,6-10,4 en hombre; y de 10,1-11 en mujer) o grande $(<9,6$ en hombre $y<10,1$ en mujer), los adolescentes de ambos sexos y provincias presentaban una complexión mediana. Sí se ha observado que los adolescentes de la provincia de Badajoz presentaban mayores valores para los parámetros relacionados con la musculatura; mientras que en los de Cáceres, los valores para parámetros relacionados con la grasa corporal eran más elevados. Esas pequeñas diferencias podrían ser indicativas que hay un mayor desarrollo muscular en los alumnos de Badajoz, mientras que los de Cáceres presentarían mayores depósitos de grasa. Sin embargo, sería necesario corroborar estos resultados con estudios posteriores, ya que no se han estudiado las posibles diferencias socioeconómicas entre ambas provincias.

Al comparar por tamaños de población, en general, los alumnos de las poblaciones rurales tenían un menor 
Tabla 4. Comparación de prevalencia de sobrepeso y obesidad en adolescentes, por sexo y tamaño de municipio de procedencia, utilizando diferentes criterios.

\begin{tabular}{|c|c|c|c|c|c|c|c|}
\hline & \multirow{2}{*}{$\begin{array}{c}\text { Grupos de Población } \\
\text { (nº habitantes) }\end{array}$} & \multicolumn{3}{|c|}{ Sobrepeso \% (IC95\%) } & \multicolumn{2}{|c|}{ Obesidad \% (IC95\%) } & \multirow[b]{2}{*}{ Media } \\
\hline & & Chicas & Chicos & Media & Chicas & Chicos & \\
\hline \multirow[t]{5}{*}{ IOTF ${ }^{17}$} & $<5.000$ & $21,7(20,8-22,6)$ & $22,1(21,2-23,0)$ & $21,8(20,9-22,7)$ & $1,7(1,4-2,0)$ & $4,4(4,0-4,8)$ & $2,7(2,4-3,0)$ \\
\hline & $5.000-10.000$ & $23,6(22,7-24,6)$ & $23,4(22,5-24,4)$ & $23,5(22,6-24,5)$ & $10,1(9,5-10,7)$ & $1,6(1,4-1,9)$ & $6,5(6,0-7,0)$ \\
\hline & $10.000-20.000$ & $17,0(16,2-18,1)$ & $29,6(28,5-30,7)$ & $23,4(22,5-24,4)$ & $3,8(3,4-4,2)$ & $1,9(1,6-2,2)$ & $2,8(2,5-3,1)$ \\
\hline & $20.000-60.000$ & $28,0(27,0-29,0)$ & $24,4(23,4-25,4)$ & $26,1(25,1-27,1)$ & $8,0(7,5-8,6)$ & $5,1(4,7-5,5)$ & $6,5(6,0-7,0)$ \\
\hline & $>60.000$ & $27,4(26,4-28,4)$ & $24,5(23,5-25,5)$ & $26,0(25,0-27,0)$ & $12,4(11,7-13,1)$ & $14,7(14,0-15,5)$ & $13,5(12,8-14,2)$ \\
\hline CDC & $<5.000$ & $13,3(13,0-14,0)$ & $11,8(11,1-12,5)$ & $12,8(12,1-13,5)$ & $2,5(2,2-2,8)$ & $8,8(8,2-9,4)$ & $4,8(4,4-5,2)$ \\
\hline \multirow[t]{4}{*}{ Growth Charts ${ }^{18}$} & $5.000-10.000$ & $20,2(19,3-20,9)$ & $14,1(13,4-14,9)$ & $17,6(16,8-18,4)$ & $7,9(7,4-8,5)$ & $7,8(7,3-8,4)$ & $7,8(7,3-8,4)$ \\
\hline & $10.000-20.000$ & $15,1(14,3-15,9)$ & $18,5(17,7-19,3)$ & $16,8(16,0-17,6)$ & $3,8(3,4-4,2)$ & $7,4(6,9-7,9)$ & $5,6(5,1-6,1)$ \\
\hline & $20.000-60.000$ & $18,7(17,9-19,4)$ & $14,1(13,4-14,8)$ & $16,3(15,5-17,1)$ & $8,0(7,5-8,6)$ & $11,5(10,8-12,2)$ & $9,8(9,2-10,4)$ \\
\hline & $>60.000$ & $22,1(21,2-22,9)$ & $14,7(14,0-15,5)$ & $18,6(17,8-19,5)$ & $10,6(10,0-11,2)$ & $19,6(18,7-20,5)$ & $14,9(14,1-15,7)$ \\
\hline \multirow[t]{5}{*}{ Sobradillo ${ }^{19}$} & $<5.000$ & $13,3(12,6-13,6)$ & $2,9(2,6-3,2)$ & $9,6(9,0-10,2)$ & $10,8(10,2-11,4)$ & $13,2(12,5-13,9)$ & $11,7(11,0-12,4)$ \\
\hline & $5.000-10.000$ & $14,6(13,9-15,1)$ & $6,3(5,8-6,8)$ & $11,1(10,5-11,8)$ & $21,3(20,4-22,2)$ & $6,3(5,8-6,8)$ & $15,0(14,2-15,8)$ \\
\hline & $10.000-20.000$ & $22,6(21,7-23,3)$ & $11,1(10,5-11,8)$ & $16,8(16,0-17,6)$ & $7,5(7,0-8,0)$ & $7,4(6,9-7,9)$ & $7,5(7,0-8,0)$ \\
\hline & $20.000-60.000$ & $14,7(14,0-15,3)$ & $9,0(8,4-9,6)$ & $11,8(11,1-12,5)$ & $21,3(20,4-22,2)$ & $14,1(13,4-14,8)$ & $17,6(16,8-18,4)$ \\
\hline & $>60.000$ & $17,7(16,9-18,3)$ & $10,8(10,2-11,4)$ & $14,4(13,7-15,1)$ & $25,7(24,7-26,7)$ & $21,6(20,7-22,5)$ & $23,7(22,8-24,7)$ \\
\hline
\end{tabular}

desarrollo corporal que los de municipios urbanos. De forma general, las chicas de localidades rurales presentarían un menor desarrollo que las del resto de municipios, considerados urbanos. Sin embargo, los chicos tendrían características corporales similares independientemente del tipo de localidad de procedencia. Hay una gran cantidad de literatura que evalúa el estado nutricional de niños y adolescentes en localidades de gran tamaño (mayores de 60.000 habitantes). Comparando los resultados de los parámetros antropométricos de los adolescentes de Extremadura con los de otras ciudades españolas, se encontró que éstos presentaban una altura inferior a la de los adolescentes de Leganés y Barcelona de la misma edad y sexo ${ }^{26,27}$. En el estudio realizado con adolescentes de Leganés de 14-15 años, la altura fue de $171 \mathrm{~cm}$ para los chicos y $161 \mathrm{~cm}$ para las chicas ${ }^{27}$, ambas superiores a las de nuestros alumnos de 14-15 años. En cuanto al peso, en casi todos los casos, los adolescentes extremeños presentaban un peso medio mayor que los de Barcelona ${ }^{26}$; en el estudio de Leganés ${ }^{27}$, realizado con adolescentes de 14 y 15 años, los chicos tuvieron un peso medio de $60,23 \mathrm{~kg}$, mayor al de los chicos extremeños; mientras que el peso de las chicas (de 58,73) fue menor que el correspondiente a las adolescentes de Extremadura.

Para el pliegue cutáneo tricipital, estudios de adolescentes de Madrid y Cádiz ${ }^{16,28}$, presentaban valores medios menores a los chicos y chicas extremeños para las mismas edades. También el IMC de los adolescentes del presente estudio fue mayor, en casi todas las edades y en ambos sexos, que el de adolescentes de Leganés, Barcelona, Madrid y Cádiz ${ }^{16,26,27,28}$. En lo que respecta a otros parámetros antropométricos relacionados con el área muscular y grasa del brazo, los adolescentes extremeños (tanto chicos como chicas) presentaban una menor área grasa del brazo que en los estudios realizados en Leganés ${ }^{27}$. Sin embargo, el área grasa del brazo de los adolescentes fue mayor a todas las edades estudiadas que la de los adolescentes de Madrid $^{16}$. En este último estudio, el área muscular del brazo de los chicos fue superior al de los extremeños para la mayoría de las edades; mientras que las chicas mostraban una mayor variabilidad en función de la edad. En lo que respecta al área total del brazo y al perímetro del brazo, los adolescentes extremeños mostraron en la mayoría de las edades valores inferiores a los de Madrid. Según lo anterior, los adolescentes de poblaciones urbanas de más de 60.000 habitantes de Extremadura parecen tener un menor desarrollo y una mayor tendencia al sobrepeso que en otras ciudades de características similares.

Como ya se ha indicado, existe una gran controversia en lo que respecta a los criterios de referencia para definir 
sobrepeso y obesidad en adolescentes. Para este estudio se utilizaron tres criterios internacionales y nacionales ${ }^{17,18,19}$, que nos permitieron comparar los resultados obtenidos con los de otros autores. En el presente estudio, dependiendo del criterio de corte del autor consultado se encontraron variaciones importantes en los porcentajes de sobrepeso y de obesidad; y además, no hubo consenso, en función del criterio escogido, a la hora de decidir el estrato de población con un menor porcentaje de obesidad. Las diferencias se vieron más claramente al realizar el sumatorio de sobrepeso y obesidad, a lo que se denominó exceso de peso. En este caso, las localidades de más de 60.000 habitantes presentaban los mayores porcentajes medios de exceso de peso, y las localidades rurales los menores. Analizando los resultados por sexos, en todos los casos, tanto las chicas como los chicos de poblaciones urbanas de más de 60.000 habitantes presentaban los mayores porcentajes generales de exceso de peso.

Los resultados obtenidos en este estudio contrastan con los de otros autores; Coronado y cols..$^{15}$ observaron un mayor porcentaje de prevalencia y obesidad de escolares de 6 a 14 años de localidades rurales de menos de 5.000 habitantes, al compararlas con otras mayores. Resultados que coinciden con los de Sánchez-Cruz y cols. ${ }^{12}$, para niños y adolescentes de 8 a 17 años y poblaciones de menos de 10.000 habitantes. Estudios realizados en otros países también muestran un mayor riesgo de sobrepeso y obesidad en chicos en edad escolar pertenecientes a zonas rurales.

Bertoncello y cols. ${ }^{29}$ indican que los chicos en edad escolar de áreas rurales de Italia tenían un mayor riesgo de sobrepeso y obesidad a los de áreas urbanas. En estudios finlandeses y canadienses ${ }^{30,31}$, también observaron que el sobrepeso era más común en zonas rurales que en áreas urbanas. Sin embargo, los resultados de Martínez-López y Redecillas ${ }^{32}$ coinciden con los del presente estudio, encontrando mayores índices de sobrepeso y obesidad en niños de 6 a 12 de hábitats urbanos que los correspondientes de zonas rurales. Por otra parte, en cuanto a las diferencias entre chicos y chicas, Rodríguez y cols. ${ }^{27}$, analizando una población de adolescentes de $3^{\circ}$ de la ESO (14 y 15 años) de la ciudad de Leganés, encontraron un menor porcentaje de chicos con un IMC normal o normopeso que en chicas, habiendo un mayor porcentaje de chicos obesos.

\section{CONCLUSIONES}

Apenas se han encontrado diferencias significativas en los parámetros antropométricos de los adolescentes de las provincias extremeñas. Al analizar por sexos, los chicos de la provincia de Badajoz presentaban un mayor desarrollo muscular. Por tamaños de localidad, los alumnos de municipios rurales presentaban los valores más bajos en la talla, peso, perímetro del brazo, área total del brazo, área muscular y área grasa del brazo. Esto nos lleva a considerar que en las localidades urbanas el desarrollo corporal en los adolescentes es más acelerado que en los municipios rurales de Extremadura. Al comparar en función del tamaño de población y sexo, se encontraron diferencias entre chicos y chicas dependiendo del tamaño de la población de origen. En general, parece existir una mayor variabilidad en la estructura y composición corporal de las alumnas dependiendo del tamaño de la localidad de procedencia. En los chicos hubo una mayor homogeneidad en esos parámetros. Al analizar los resultados de sobrepeso y obesidad se han observado diferencias en función del tamaño del municipio y el sexo, que dependieron del criterio de referencia escogido. Al valorar el exceso de peso en general, se observó una mayor prevalencia en las localidades de mayor tamaño y una menor prevalencia en las rurales. Las principales limitaciones de este estudio son las inherentes a su carácter transversal. Aunque los resultados obtenidos no pueden ser generalizados, son muy relevantes, ya que fueron obtenidos de modo estratificado y pueden ser utilizados en otros estudios a nivel nacional e internacional. Sin embargo, para confirmar las conclusiones obtenidas y determinar la evolución de la prevalencia de exceso de peso en localidades rurales y urbanas, sería necesario realizar otros estudios en años posteriores.

\section{Conflicto de intereses. Los autores de este estudio declaran no tener ningún conflicto de intereses}

Agradecimientos. Este trabajo ha sido financiado por la Junta de Extremadura y el Fondo Social Europeo (ref. GR15166). Los autores de este estudio desean agradecer a la Junta de Extremadura y a los centros educativos de la Comunidad de Extremadura por su apoyo para el desarrollo del presente trabajo.

\section{BIBLIOGRAFÍA}

1. Serra L, Ribas L, Aranceta J, Pérez C, Saavedra P, Peña L. Childhood and adolescent obesity in Spain. results of the ENKid study (1998-2000). Med Clin (Barc) 2003; 121(19): 725-732.

2. Grao-Cruces A, Nuviala A, Fernández-Martínez A, PorcelGálvez AM, Moral-García JE, Martínez-López EJ. Adherence to Mediterranean diet in rural urban adolescents of southern Spain, life satisfaction, anthropometry, and physical and sedentary activities. Nutr Hosp 2013; 28(4): 1129-1135.

3. De-Rufino PM, Antolín O, Casuso I, Mico C, Amigo T, Noriega MJ, Santamaría A, Sobaler S, Jaen P, Carrasco M, Salcines $R$, Rivero LA, Redondo C. Assessment of nutritional risk among in-school adolescents from Cantabria. Nutr Hosp 2014; 29(3): 652-657.

4. Wang Y, Lobstein T. Worldwide trends in childhood overweight and obesity. Int J Pediatr Obes 2006; 1: 11-25.

5. Lobstein T, Baur L, Uauy R. Obesity in children and young people. IASO. Obes Rev 2004; 5(1): S4-85.

6. Gordon-Larsen P, The NS, Adair LS. Longitudinal Trends in Obesity in the United States From Adolescence to the Third Decade of Life. Obes (Silver Spring) 2010; 18(9): 1801-1804.

7. Enes CC, Slater B. Obesity in adolescence and its main determinants. Rev Bras de Epidemiol 2010; 13(1): 163-171.

8. Moreno LA, Mesana MI, Fleta J, Ruiz JR, González-Gross M, Sarría A, Marcos A, Bueno M. Overweight, obesity and body fat composition in Spanish adolescents. The AVENA Study. 
Ann Nutr Metab 2005; 49: 71-76.

9. Serra-Majem L, Bartrina JA, Pérez-Rodrigo C, Ribas-Barba L, Delgado-Rubio A. Prevalence and determinants of obesity in Spanish children and young people. Br J Nutr 2006; 96(S1): S67-S72.

10. Henríquez $P$, Doreste I, Laínez P, Estévez MD, Iglesias $M$, Martín G, Sosa I, Serra-Majem L. Prevalence of obesity and overweight in adolescents from Canary Islands, Spain. Relationship with breakfast and physical activity. Med Clin (Barc) 2008; 130(16): 606-610.

11. Aranceta-Bartrina J, Serra-Majem L, Foz-Sala M, MorenoEsteban B. Prevalence of obesity in Spain. Med Clin (Barc) 2005; 125(12): 460-466.

12. Sánchez-Cruz JJ, Jiménez-Moleón JJ, Fernández-Quesada F, Sánchez MJ. Prevalence of Child and Youth Obesity in Spain in 2012. Rev Esp Cardiol 2013; 66(5): 371-376.

13. MSSI. ENSE Encuesta Nacional de Salud España 2017. Ministerio de Sanidad, Consumo y Bienestar Social, 2018. (Consultado el 2/07/2018) Disponible en: https://www.msssi.gob.es/ estadEstudios/estadisticas/sis/nfSanSNS/UltDatos.htm.

14. López-Azpiazu I, Sánchez-Villegas A, Johansson L, Petkeviciene J, Prattala R, Martínez-González MA. Disparities in food habits in Europe: systematic review of educational and occupational differences in the intake of fat. J Hum Nutr Diet 2003; 16(5): 349-364.

15. Coronado V, Odero D, Canalejo D, Cidoncha J. Prevalence of overweight and obesity in schoolchildren in rural areas. Gac Sanit [online] 2012; 26 (5): 460-462.

16. Marrodán MD, Callejo ML, Moreno-Heras E, GonzálezMontero de Espinosa M, Mesa PM, Gordón PM, García FF. Nutritional anthropometry and physical performance in urban adolescents of Madrid. An Esp Pediatr 1999; 51(1): 9-15.

17. Cole TJ, Bellizzi MC, Flegal KM, Dietz WH. Establishing a standard definition for child overweight and obesity worldwide: international survey. BMJ 2000; 320(7244): 1240-1243.

18. Kuczmarski RJ, Ogden CL, Guo SS, Grummer-Strawn LM, Flegal KM, Mei Z, Wei R, Curtin LR, Roche AF, Johnson $C L$. 2000 CDC growth charts for the United States: Methods and development. National Center for Health Statistics. Vital Health Stat 2002; 11(246).

19. Sobradillo B, Aguirre A, Aresti U, Bilbao A, Fernández-Ramos C, Lizárraga A, Lorenzo H, Madariaga L, Rica I, Ruiz I, Sánchez E, Santamaría C, Serrano JM, Zabala A, Zurimendi B, Hernández $M$. Spanish growth reference curves and charts. Longitudinal and Transversal studies. Bilbao: Fundación Faustino Orbegozo, Bilbao, ISBN 84-607-9967-0, 2004; 1-31.
20. MARM. Population and rural society. Analysis and prospective - Serie Agrlnfo no12. Subdirección General de Análisis, Prospectiva y Coordinación, Subsecretaría. Ministerio de Medio Ambiente y Medio Rural y Marino, 2009. (Consultado el 15/11/2016) Disponible en: http://www.mapama.gob.es/es/ ministerio/servicios/analisis-y-prospectiva/Agrinfo12_tcm7161562.pdf

21. Malina RM. The measurement of body composition. In: Johnston FE, Roche AF, Susanne CH, editors. Human Physical Growth and Maturation. New York, Plenum Press, 1980; pp. 59-89.

22. Weiner JS, Lourie JA. Practical human biology. Londres: Academic Press, 1981.

23. Fernández I, Aranda E, Córdoba MG, Hernández A, Rodríguez JA, Pérez-Nevado F. Nutritional evaluation of adolescent students from Extremadura based on anthropometric measurements. Nutr Hosp 2014; 29(3): 665-673.

24. Bryman A, Cramer D. Quantitative data analysis with IBM SPSS 17, 18 \& 19: A guide for social scientists. Routledge, 2012.

25. Mataix J. Food and human nutrition. Ed Ergón. Majadahonda. Madrid, 2002.

26. Carrascosa A, Yeste D, Copil A, Gussinyé M. Secular growth changes. weight, height and body mass index values in infant, children, adolescent and young adults from Barcelona population. Med Clin (Barc) 2004; 123(12): 445-451.

27. Rodríguez M, García A, Salinero JJ, Pérez B, Sánchez JJ, Gracia $R$, Robledo $S$, Ibáñez $R$. Diet quality and its relation to sex and BMI in adolescents. Nutr Clin Diet Hosp 2012; 32(2).

28. Ruiz-Jiménez MA, Fernández JR, Pavón R. Anthropometric assessment of the nutritional state of the adolescent population of Cadiz. An Esp Pediatr 1996; 45(4): 369-376.

29. Bertoncello C, Cazzaro R, Ferraresso A, Mazzer R, Moretti G. Prevalence of overweight and obesity among school-aged children in urban, rural and mountain areas of the Veneto Region, Italy. Public Health Nutr 2008; 11: 887-890.

30. Ismailov RM, Leatherdale ST. Rural-urban differences in overweight and obesity among a large sample of adolescents in Ontario. Int J Pediatr Obes 2010; 5: 351-360.

31. Vuorela N, Saha MT, Salo M. Prevalence of overweight and obesity in 5- and 12-year-old Finnish children in 1986 and 2006. Acta Paediatr 2009; 98: 507-512.

32. Martínez-López EJ, Redecillas-Peiró MT. Prevalence of overweight and obesity in school children in the province of Jaen. Rev Int Med Cienc Act Fis Deporte 2011; 11(43): 472-490. 